\title{
¿PUEDE UNA NUEVA CONSTITUCIÓN FORTALECER LA RESPUESTA DEL ESTADO ANTE LAS EMERGENCIAS?
}

Bárbara Sepúlveda Hales y Lieta Vivaldi Macho 


\section{BÁRBARA SEPÚLVEDA HALES}

Magíster en Derecho Público de la Universidad de Chile y Magíster en Género de la London School of Economics and Political Science. Abogada de la Universidad de Chile. Es autora del libro Género $y$ derecho. Es profesora de las cátedras de Derecho Constitucional y Teoría Feminista del Derecho en la Universidad Alberto Hurtado, y profesora invitada en diversos cursos de pre y postgrado de la Facultad de Derecho de la Universidad de Chile. Se especializa en género y derecho y en derecho público. Es cofundadora y directora ejecutiva de la Asociación de Abogadas Feministas, Abofem. Correo electrónico: basepulveda@uchile.cl.

\section{LIETA VIVALDI MACHO}

Doctora en Sociología de Goldsmiths, University of London, Magíster en Sociología de la London School of Economics and Political Science, diplomada en género y violencia de la Facultad de Filosofía de la Universidad de Chile y abogada de la Universidad de Chile. Actualmente dirige el programa de Género y Derecho en la Facultad de Derecho de la Universidad Alberto Hurtado, trabaja como investigadora en el Centro de Ética Aplicada (Cedea) de la Universidad de Chile y como investigadora asociada de la Facultad de Derecho de la Universidad Diego Portales. Es coordinadora de la línea biopolítica y secretaria del International Institute for Philosophy and Social Studies (Santiago, Chile), forma parte del directorio de RED y de Abofem. Se especializa y hace clases sobre derechos humanos, derechos sexuales y reproductivos, sociología jurídica, biopolítica y feminismos.

Correo electrónico: lieta.vivaldi@uchile.cl 


\section{¿PUEDE UNA NUEVA CONSTITUCIÓN FORTALECER LA RESPUESTA DEL ESTADO ANTE LAS EMERGENCIAS?}

La pandemia del Covid-19, además de ser una crisis sanitaria, afecta distintos aspectos de nuestro sistema económico y social. La situación de vulneración que ya viven ciertos grupos se ve exacerbada en un contexto de crisis y son los grupos más vulnerables (considerando las variables de género, grupos racializados, edad, clase social, entre otros) los que sufren las consecuencias más dramáticas. La respuesta oportuna y efectiva desde el Estado para adoptar e implementar medidas que controlen la pandemia es de una importancia decisiva.

En el actual contexto recrudece la violencia en casi todos sus niveles, y al hablar de violencia en Chile reaparece con mayor fuerza ese fantasma con el que hemos aprendido a vivir: la desigualdad estructural. La desigualdad es uno de los factores determinantes de la violencia y a la vez es violencia en sí misma. En todas las radiografías sociales de la violencia encontramos como factor común la desigualdad basada en jerarquías de clase, raza, edad y género. Desde su nivel simbólico (y jurídico), el problema de la desigualdad gira en torno a un centro que determina quién queda en cuál escalón de esa jerarquía social, dependiendo de cuánto se ajuste a la norma, al modelo que determina quién tiene derechos, quién tiene poder, quién es realmente libre.

Desde el feminismo se ha analizado y criticado el parámetro androcéntrico del derecho, su universalismo y pretendida pero falsa igualdad (Olsen, 1990; Smart, 1992; Zúñiga 2009). Este parámetro es el mismo que encontramos tras múltiples instituciones sociales: el hombre de raza blanca, heterosexual, con poder adquisitivo, sin discapacidades, con familia y patrimonio. Vemos entonces cómo ese parámetro va fijando los criterios de pertenencia y de reconocimiento y, al mismo tiempo va determinando a los excluidos, los de segundo orden de importancia. Si pensamos en los segmentos de la población altamente vulnerables a sufrir las peores consecuencias de esta crisis nos encontramos con la población carcelaria, las mujeres, las y los trabajadores informales, las personas en situación de discapacidad, migrantes, las personas racializadas, la población LGBTIQ, las niñas, niños y adolescentes; es decir, lo que vemos es la otredad en carne y hueso.

En este sentido, hoy solo se nos hace más evidente algo que constituye a nuestras sociedades "democráticas", y aunque no hay nada de nuevo en esas realidades, nos sorprende y estremece un profundo temor a la pobreza, al incremento de la desigualdad, a la cruda verdad de nuestras jerarquías construidas bajo el imperio de un Estado de derecho inspirado en los principios de igualdad, libertad y fraternidad, 
y bajo un modelo económico que auguraba la autorregulación del mercado, la mano invisible y el libre intercambio como manifestaciones máximas de la autonomía, bienestar, la riqueza y la libertad de las personas. Lo que hoy vivimos en Chile es que ambos modelos, Estado-gobierno (el modelo democrático) y economía neoliberal (el modelo productivo), entraron en crisis conjuntamente.

En este artículo veremos, en primer lugar, los estados de excepción constitucional para analizar cómo han sido utilizados por el gobierno de Chile en la pandemia derivada del Covid-19. Luego analizaremos el Estado neoliberal de derecho y su incapacidad de dar respuestas adecuadas que otorguen bienestar y derechos mínimos a las personas. Defenderemos la idea de un Estado social de derecho como una alternativa para garantizar una vida digna y más justa. Por último, analizaremos la violencia de género en la pandemia y el deber del Estado de prevenir, sancionar y erradicar.

\section{LOS ESTADOS DE EXCEPCIÓN: CÓMO ENFRENTAR LA PANDEMIA}

El miércoles 18 de marzo de 2020, el presidente Sebastián Piñera declaró Estado de Excepción Constitucional de Catástrofe ante la emergencia por Coronavirus Covid-19. Este Estado de Excepción está regulado en la Constitución (artículos 39 a 45) y en la Ley Orgánica Constitucional Nº18.415.

En caso de emergencias naturales, calamidades públicas, los estados de excepción son muy diferentes a los estados de excepción constitucional que se utilizan para el control social, ya que entregan mayores herramientas que puedan atender las consecuencias económicas de una catástrofe natural. El presidente tiene las facultades de restringir las libertades de locomoción y de reunión; disponer requisiciones de bienes; establecer limitaciones al ejercicio del derecho de propiedad y adoptar todas las medidas extraordinarias de carácter administrativo que sean necesarias para el pronto restablecimiento de la normalidad en la zona afectada (artículos 41 y 43 de la Constitución Política de la República de Chile). El presidente y autoridades, de este modo, pueden hacerse cargo de que los bienes esenciales lleguen a todas las personas, incluso tienen la potestad de intervenir sus precios. Hemos visto, sin embargo, que en la pandemia actual se han utilizado solo las atribuciones militarizadas del uso de la fuerza de este estado de excepción. Es decir, aun existiendo un amplio margen que esta herramienta le otorga al presidente de la República para invocar restricciones al derecho de propiedad y tener un mayor control económico, solo se han utilizado aquellas medidas que permiten actuar excepcionalmente mediante el toque de queda y el incremento de la seguridad y el orden a través de las Fuerzas de Orden y las Fuerzas Armadas.

Cabe recordar que el 18-O el presidente Piñera decretó Estado de Excepción Constitucional de Emergencia que, de acuerdo a la Constitución, puede decretarse 
en caso de grave alteración del orden público o de grave daño para la seguridad de la nación (artículo 42). Sabido es que lo anterior resultó en una serie de violaciones de derechos humanos. Amnistía Internacional, en su informe "Los derechos humanos en las Américas: retrospectiva 2019”, al analizar la situación del 18-O señaló que "Chile cerró el 2019 con la peor crisis de derechos humanos, desde el régimen del general Augusto Pinochet" (Soy Chile, 2020). Las manifestaciones fueron reprimidas con fuerza por parte del Estado y, siguiendo lo señalado en el informe, "intentaron justificar su uso de la violencia contra manifestantes, alegando que tales medidas eran necesarias para proteger las infraestructuras y la propiedad privada frente a daños y vandalismo". Durante el Estado de Excepción Constitucional de Emergencia aumentó el número de abusos cometidos por el Estado: perdieron la vida 31 personas, al menos cuatro de ellas a manos de fuerzas estatales.

En específico, la violencia sexual por parte de agentes del Estado contra mujeres y disidencia sexual fue recurrente, tal como lo fue la desidia institucional en la defensa de mujeres víctimas (Sepúlveda y Vivaldi, 2019).

Un estado de excepción, para poder operar "democráticamente" debe, en primer lugar, limitar el poder presidencial unilateral. El o la presidente/a debería poder declarar solo por una semana o dos la excepción y dejar en manos del Congreso la decisión. Los poderes para el presidente deberían depender de la voluntad del Congreso y, aun así, por un tiempo máximo determinado, cuya extensión debería requerir mayores quórum. Esto garantiza su real excepcionalidad y se previenen vulneraciones de derechos (Hunter, 2009).

La Corte Interamericana de Derechos Humanos, por su parte, hizo un llamado a que los Estados aseguren que todas las medidas que tomen en relación a la pandemia estén de acuerdo a sus compromisos internacionales. Especificó que

Cualquier restricción o suspensión adoptada debe tener sustento en la mejor evidencia científica y considerar, de manera previa a su adopción y durante su implementación, los particulares efectos que puede tener sobre los grupos más vulnerables con el fin de asegurar que su impacto no sea especialmente desproporcionado mediante la adopción de las medidas positivas que resulten necesarias (OEA, 2020).

\section{HACIA UN ESTADO SOCIAL DE DERECHO}

El Estado neoliberal de derecho ha demostrado ser un marco jurídico deficiente a la hora de asegurar un mínimo de bienestar general a las personas. El gobierno, por un lado, ha abusado de sus atribuciones constitucionales hiperpresidencialistas 
para poner urgencia a los proyectos de ley de su agenda de seguridad e inteligencia antiprotestas y ha frenado las iniciativas por mociones que puedan ampliar el acceso a prestaciones sociales, derechos laborales, entre otras.

Lo anterior se relaciona con el modelo implementado en la Constitución de 1980, el que atribuye excesivas facultades al o la presidente de la República, consagra un sistema económico neoliberal que prioriza la actuación y libertad de los llamados "grupos intermedios" por sobre el Estado, no garantiza los derechos económicos, sociales y culturales y, en aras de asegurar una "igualdad formal", profundiza desigualdades, ya que no consagra derechos específicos para personas de grupos desaventajados. La actual Constitución no garantiza los derechos económicos sociales y culturales y consagra un Estado subsidiario. En Chile, además, se ha entendido un Estado subsidiario en que el mercado tiene la prioridad en la provisión de los derechos, mientras que el Estado no asume ninguna responsabilidad con la ciudadanía. Estos aspectos impactan, sin duda, en los sectores más vulnerables de la población y, por lo tanto, en este contexto de pandemia se agudizan los efectos negativos de la desigualdad que viven en Chile la población carcelaria, las mujeres, las y los trabajadores informales, las personas en situación de discapacidad, los migrantes, las personas racializadas y la población LGBTIQ.

La necesidad de cambiar la Constitución es evidente. En una reciente encuesta, realizada en alianza entre Humanas, el Observatorio de Género y Equidad y Tuinfluyes. com, un 74 por ciento de las mujeres señaló que aprueba una nueva Constitución. Respecto al sistema político, un 62 por ciento se inclina por uno semipresidencial, donde exista mayor equilibrio entre presidente/a y Parlamento y un 77 por ciento cree que debería haber solo una cámara legislativa. Otro tema relevante es la pérdida de confianza en las instituciones. Según el Índice de Percepción de la Democracia $2020^{1}$, para el 73 por ciento de los chilenos la democracia es importante, pero solo un 42 por ciento considera que el país es democrático versus un 51 por ciento que lo consideró así el 2019. Asimismo, Chile tiene la segunda mayor proporción de personas en 2020 (72 por ciento) que perciben que su gobierno generalmente actúa en interés de un pequeño grupo de personas.

Desde el 18-O, la revuelta popular ya había puesto al descubierto las profundas secuelas del modelo neoliberal: los derechos a la salud, educación, a una vida libre de violencia y en un medio ambiente sano (asociado a derechos a alimentación, agua, aire), entre tantos otros, más que derechos, son privilegios de algunos/as. La pandemia, entonces, ha venido a reafirmar la profunda desigualdad que existe

1. Estudio anual realizado por tercera vez por la encuestadora alemana Dalia Research y la Alliance of Democracies Foundation (Fuentes, 2020). 
en nuestro país y la necesidad de cambiar el ya insostenible modelo impuesto por unos pocos en 1980. El neoliberalismo, como sistema gubernamental, aplica y generaliza aquellos principios económicos y del mercado a la totalidad de las esferas de la vida (Brown, 2015; Foucault, 2008). El Estado interviene solo para asegurar que las condiciones del libre mercado se mantengan. Por ello no es raro que la Constitución de 1980 asegure libertades y no derechos. Ejemplos son la libertad de elegir plan de salud, pero no el derecho a la salud, y lo mismo en la educación, donde lo único asegurado es la libertad de elegir establecimiento educacional, pero no el derecho a la educación laica, no sexista y de calidad, como han pedido los movimientos sociales. En ambos ámbitos, por lo demás, es una libertad supeditada al poder adquisitivo de quien "decide", por lo que no es libertad propiamente tal. El neoliberalismo, además, se sustenta en la competencia entre individuos, por lo que los tejidos sociales fueron disueltos y se incentivó la cultura egoísta e individualista. El 18-O mostró una rearticulación de las organizaciones barriales y comunitarias a través de cabildos y asambleas que han sido clave para enfrentar esta pandemia en comunidad (Biskupovic, 2020). Las ollas comunes, las respuestas desde las organizaciones que generan redes de apoyo y contención son muestras de cómo el sentido de lo colectivo es una necesaria respuesta a la crisis, pero se necesita de una respuesta también articulada desde el propio Estado.

Por otra parte, diversas leyes que servirían para otorgar derechos esenciales a las personas durante la pandemia han sido cuestionadas por su inconstitucionalidad. Una minuta del gobierno establece que son 46 (19 en el Senado y 27 en la Cámara). La mayoría de ellas han sido presentadas este año en el marco de la pandemia por el Covid-19 (La Tercera, 2020). Ejemplos de leyes que durante la pandemia se han considerado inconstitucionales son: postnatal de emergencia, suspensión de deudas CAE, el proyecto que prohíbe el corte de servicios básicos, el que propone el retiro de fondos parciales de las AFP y el que establece pensiones básicas solidarias, iniciativa de cobro de impuestos a los súper ricos para financiar una renta básica de emergencia. Todos estos proyectos están destinados a otorgar derechos a personas en contexto de crisis y aun así la autoridad ha tratado de impugnarlos por considerarlos contrarios a la Constitución.

Además de ejemplos en este contexto, el argumento de inconstitucionalidad ha sido usado en variadas ocasiones en que se ha intentado amortiguar o cambiar en algo el modelo neoliberal. Es decir, lo anterior no es algo propio de la pandemia; obstruir o cambiar el sentido de leyes tendientes a otorgar derechos económicos sociales y culturales a las personas o garantizar una mayor igualdad han sido acciones permanentes a través del recurso de inconstitucionalidad. En el caso de la Ley 19.130 de interrupción voluntaria del embarazo en tres causales, al pasar por el Tribunal Constitucional fue ampliada la objeción de conciencia de personal a 
institucional, lo que privilegió la libertad de los grupos intermedios por sobre los derechos de las mujeres (Undurraga y Sadler, 2019).

Como hemos visto, la actual Constitución que establece un Estado neoliberal de derecho no da las respuestas que se necesitan para una sociedad más justa y que permita desarrollar los proyectos vitales personales y colectivos. En este sentido, es interesante ver el reciente pronunciamiento de la Cepal sobre las medidas de políticas sugeridas para enfrentar la crisis, donde concluye que las estrategias que adopten los países a mediano y largo plazo deben conducir a un Estado de bienestar con protección social universal2 ${ }^{2}$ La actual Constitución de 1980, en cambio, y como señalamos, consagra un modelo neoliberal con todas sus implicancias ya esbozadas.

Por ello estamos en un momento crucial en que surge la pregunta por el rol que debiera tener el Estado. Un Estado social y democrático de derecho podría otorgarnos un marco jurídico mucho más amplio para enfrentar una crisis sanitaria y sus ribetes sociales, que hoy se convierten en las consecuencias más graves para la mayoría de la población. Esto implica superar la simple barrera del cambio constitucional e ir más allá con el objetivo de redefinir el pacto social. Un Estado social y solidario de derecho implica que la institucionalidad pueda usar los recursos públicos para mejorar sus servicios públicos, a los que deberíamos aportar según nuestras posibilidades y recibir de acuerdo a nuestras necesidades. Como figura política, el Estado social "supone que los poderes públicos (principalmente la Administración) asumen la responsabilidad de otorgar a la generalidad de los ciudadanos las prestaciones y servicios adecuados para la satisfacción de sus necesidades vitales" (Viera, 2014). Siguiendo a García-Pelayo, "mientras que en los siglos XVIII y XIX se pensaba que la libertad era una exigencia de la dignidad humana, ahora se piensa que la dignidad humana (materializada en supuestos socioeconómicos) es una condición para el ejercicio de la libertad" (citado en Viera, 2014). Si pensamos en el elocuente lema "hasta que la dignidad se haga costumbre" nos damos cuenta de que sin condiciones materiales mínimas esto no es posible, por ello el Estado debe garantizarlas.

2. Comisión Económica para América Latina y el Caribe (Cepal), Informe sobre el impacto económico en América Latina y el Caribe de la enfermedad por Coronavirus (Covid-19): estudio elaborado por la Comisión Económica para América Latina y el Caribe (Cepal) en respuesta a la solicitud realizada por el gobierno de México en el ejercicio de la presidencia pro témpore de la Comunidad de Estados Latinoamericanos y Caribeños (Celac) durante la Reunión Ministerial Virtual sobre Asuntos de Salud para la Atención y el Seguimiento de la Pandemia Covid-19 en América Latina y el Caribe, celebrada el 26 de marzo de 2020 (LC/TS.2020/45), Santiago, 2020. 
Diversos organismos internacionales (OMS y Cepal, por ejemplo) y los órganos de los sistemas de protección de derechos humanos han emitido pronunciamientos que realizan recomendaciones a los Estados que pertenecen a ellos. Han elaborado, de este modo, sugerencias y directrices para orientar a los países en la adopción de políticas públicas que pueden optimizar las respuestas al brote de Covid-19. Han emitido declaraciones el Comité de Derechos Económicos, Sociales y Culturales (Cdesc; UN, 2020) y el Comité de los Derechos del Niño y el Comité de Derechos Humanos (CDN, 2020; HRC, 2020). El Comité para la Eliminación de la Discriminación contra la Mujer (Cedaw), por su parte, publicó una nota de orientación para garantizar el ejercicio de los derechos de las mujeres (ONU Mujeres, 2020). En el sistema interamericano, la Comisión Interamericana de Derechos Humanos adoptó una resolución sobre pandemia y derechos humanos (Cidh, 2020).

Los organismos coinciden en la repercusión de la pandemia en los derechos económicos, sociales y culturales y dan especial énfasis a la importancia de garantizar el derecho a la salud y proteger los derechos de los/as trabajadores. Por su parte, las medidas de restricción de derechos humanos que sean adoptadas para enfrentar la pandemia deben cumplir con ciertos requisitos, como ser temporales, estrictamente necesarias y proporcionales.

Los grupos que se encuentran en especial situación de vulnerabilidad requieren medidas puntuales, por lo que se han elaborado recomendaciones especiales para ellos, entre quienes se cuentan adultos mayores, las personas privadas de libertad, las mujeres, los pueblos indígenas, las personas en situación de movilidad humana, los niños, niñas y adolescentes (NNA), las personas LGBTIQ, las personas afrodescendientes y las personas con discapacidad. En el siguiente apartado veremos algunas consideraciones específicas respecto a la pandemia y género.

\section{VIOLENCIA Y GÉNERO EN PANDEMIA: LA NECESIDAD DE PREVENIR, SANCIONAR Y ERRADICAR}

La directora ejecutiva de ONU Mujeres realizó una declaración en la que plantea su preocupación por el aumento de la violencia que era considerada ya desde antes de la pandemia como una de las violaciones más flagrantes a los derechos humanos (Mlambo-Ngcuka, 2020). En ella, resalta el rol de las organizaciones de base feministas y de mujeres, y la necesidad de encontrar un financiamiento más estable para estas entidades; a la vez, hace un fuerte llamado a los operadores judiciales y policiales para priorizar estos casos y movilizarse oportunamente. Enfatiza que el rol del Estado para proveer soluciones que permitan salir de los ambientes violentos es crucial y deben explorarse distintas estrategias para prevenir y combatir estas situaciones. En 
este sentido, para las autoridades es clave tener en cuenta que, de no implementar medidas específicas para las mujeres, la vulneración a sus derechos se acentúa.

De acuerdo a un informe reciente de Women's Link Worldwide, Amnistía Internacional y la International Planned Parenthood Federation, son tres los aspectos principales a considerar al analizar la crisis: en primer lugar, una situación excepcional no exime a los Estados de sus obligaciones reforzadas de atender las violencias basadas en género, la que también afecta a las mujeres transgénero y a las personas intersex (Ippf, 2020). Por el contrario, se exige la adopción de más y mejores medidas para minimizar los impactos de la crisis, evitando que los derechos se vean afectados. Para eliminar factores de discriminación que exacerben la vulnerabilidad, los Estados deben considerar las condiciones específicas de las niñas, adolescentes y mujeres y garantizar el acceso a prestaciones tales como salud sexual y reproductiva, protección ante la violencia y salud mental, todas ellas garantías para que, de ser necesario, desempeñen trabajos en forma segura. En este sentido, por ejemplo, es importante tener en cuenta que, en situaciones de emergencia, el acceso a prestaciones en salud sexual y reproductiva suele verse comprometido (Unfpa, 2020). Por último, las mujeres han mostrado ser clave en la toma de decisiones en el contexto de pandemia a través de la formulación de estrategias específicas, por lo que su incorporación al menos paritaria en ámbitos de decisiones debe estar considerada (Ippf, 2020).

Las condiciones reales de hacinamiento, la desigualdad y la pobreza ponen de relieve una crisis de los cuidados que repercute con mayor fuerza en las mujeres. Las cuarentenas y el cierre de establecimientos educacionales han aumentado la demanda de cuidados y tareas dentro del hogar, cargas que ya desde antes de la pandemia recaían desproporcionadamente en las mujeres (Ippf, 2020). Un sondeo realizado en abril por Cadem y la Mutual de Seguridad mostró que 56 por ciento de las mujeres sienten estar trabajando más que en tiempos normales de oficina, mientras que solo un 36 por ciento de los hombres afirmó lo mismo (El Mostrador, 2020). Solo un 14 por ciento de ellas afirmó sentir una menor carga laboral, lo que el grupo masculino cifró en 31 por ciento. Así, una de las características de esta pandemia es que ha relevado la centralidad de la tarea de cuidados, la que las mujeres continúan asumiendo en mayor medida que los hombres. Desde la economía feminista se ha venido planteando hace bastante tiempo que la disciplina económica tradicional ha olvidado sistemáticamente los trabajos que se requieren para sostener la vida: el trabajo doméstico y de cuidados es realizado mayoritariamente por mujeres y ha sido ignorado y desvalorizado tanto simbólica como materialmente (Pérez Orozco, 2014). Actualmente, esta situación tiene consecuencias muy graves.

Asimismo, las desigualdades de género se han incrementado: las mujeres son quienes desempeñan en casi un 70 por ciento las labores de salud, y las tareas de 
cuidado, como se señaló, son realizadas mayoritariamente también por mujeres (Casen, 2017; Colmed, 2020). Asimismo, la tasa de desempleo entre mujeres ha aumentado a cifras históricas y de manera más abrupta que la de los hombres (INE, 2020). Las ocupaciones más afectadas durante la crisis son los trabajos informales y precarizados, los que ocupan trabajadoras de casa particular y vendedoras de comercio, labores desarrolladas mayoritariamente por mujeres (INE, 2020).

En todo el mundo, y sin contexto de crisis o pandemia, ya el 35 por ciento de las mujeres son víctimas de violencia sexual, física o psicológica, lo que evidentemente nos alerta de que el aumento de la violencia en la pandemia puede llevarnos a cifras devastadoras. Francia, por ejemplo, recurrió al confinamiento como medida principal para luchar contra el virus, y desde el 17 de marzo, fecha en que el país entró en aislamiento definitivo, los reportes de violencia doméstica aumentaron en un 30 por ciento. En Chipre y Singapur, las líneas de emergencia por violencia de género experimentaron un aumento en las llamadas de un 30 por ciento y 33 por ciento, respectivamente. En Italia se registraron tres feminicidios durante las primeras tres semanas de la epidemia, dos de los cuales fueron perpetrados en los hogares de las víctimas. Y en Argentina, en 14 días de cuarentena por Coronavirus, se registraron 12 femicidios. Además, no podemos dejar de considerar que la violencia es y siempre ha sido multifactorial y que su origen se encuentra en una sociedad patriarcal que nos construyó como desiguales, pero que en cada caso estará cruzada por diversos factores que se entremezclan, como la pobreza, el hacinamiento, la falta de educación, el alcoholismo y la drogadicción, la misoginia o la condición de víctima de violencia que muchos agresores manifiestan. Estar recluidas en casa ha significado para muchas mujeres y niñas tener que convivir con el agresor. En Chile, en los últimos 10 días de marzo, la Unidad de Violencia contra la Mujer del Servicio Nacional de la Mujer y la Equidad de Género (Sernameg) reportó un aumento de un 125 por ciento de llamadas al teléfono de orientación en violencia contra la mujer (\#1455). La alcaldesa de la comuna de Providencia, por su parte, reportó un aumento de un 500 por ciento de los llamados por violencia de género durante la cuarentena en esa comuna (El Mostrador, 2020).

Una mirada interseccional también muestra cómo distintas mujeres experimentan los efectos de la crisis de forma diversa: no es lo mismo trabajar a distancia desde una casa cómoda que trabajar en comercio informal y verse obligada a salir, vivir con el agresor o no tener un espacio propio. Asimismo, las mujeres con algún tipo de discapacidad viven la pandemia con problemas específicos. En una investigación en curso de la University College of London (UCL) sobre la manera en que la pandemia está afectando a pobladores de zonas de bajos ingresos en países como Indonesia y Chile se evidencian los problemas específicos que la situación conlleva para mujeres que tienen algún tipo de discapacidad (Ossul-Vermehren, 2020). En 
el estudio se identifica, por una parte, la gravedad de la falta de alimento y cómo se han desarrollado cocinas comunitarias (ollas comunes, como se conocen en Chile), pero para personas con discapacidad que tienen trabajos precarios o piden dinero en las calles esto es aún más dramático. Otro punto identificado es el cuidado, que recae mayormente en mujeres. Cuando no hay redes familiares ni vecinales producto del confinamiento, para las mujeres con algún tipo de discapacidad esta situación acarrea un fuerte daño psicológico y financiero. Asimismo, las mujeres con algún tipo de discapacidad sufren violencia en proporción aún mayor (UN, 2012). Por último, es difícil acceder al agua en las poblaciones, y para las mujeres con algún tipo de discapacidad este acceso es aún más complejo.

El Estado de Chile ha contraído obligaciones internacionales a través de la suscripción de tratados y está obligado, por tanto, a cumplirlas. La Convención de Eliminación de Todas las Formas de Discriminación contra la Mujer (Cedaw) y Belém do Pará, Convención Interamericana para Prevenir, Sancionar y Erradicar la Violencia contra la Mujer, así lo establecen. En la recomendación general número 35 sobre la violencia por razón de género contra la mujer, por la que se actualiza la recomendación general número 19 de 26 de julio de 2017, se refuerzan las obligaciones de los Estados frente a la violencia de género, entre las que se cuentan el establecimiento de políticas que tiendan a eliminarla y busquen responsabilidades, por lo que deben contar con una legislación acorde. La Convención Belém do Pará, por su parte, señala en su artículo 1 que la violencia contra la mujer es "cualquier acción o conducta, basada en su género, que cause muerte, daño o sufrimiento físico, sexual o psicológico a la mujer, tanto en el ámbito público como el privado”. Asimismo, responsabiliza al Estado por la violencia contra las mujeres cuando este no ha adoptado todas las medidas adecuadas para su prevención, sanción y erradicación. De acuerdo a estos instrumentos, el Estado debe tomar las medidas legislativas pertinentes para asegurar el goce de los derechos que de ellos emanan y de esta forma cumplir su deber de protección. 


\section{REFERENCIAS}

Biskupovic, María Consuelo. Respuestas colectivas e institucionales a la pandemia [en línea]. Punto de Vista. Universidad Academia de Humanismo Cristiano, 1 de junio de 2020. <http://www.academia.cl/comunicaciones/columnas/ respuestas-colectivas-e-institucionales-a-la-pandemia-reforzar-el-trabajoterritorial> [consulta: 10 de julio de 2020].

Brown, Wendy. Undoing the Demos: Neoliberalism's Stealth Revolution. New York, Zone Book, 2015.

Casen. Región Metropolitana de Santiago, Equidad de Género: Resultados Encuesta Casen 2017. Ministerio de Desarrollo Social, documento elaborado por Santiago Gajardo. <http:/ / www.desarrollosocialyfamilia.gob.cl/storage/ docs/DOCUMENTO_EQUIDAD_DE_GENERO_RMS_CASEN_2017. pdf $>$ [consulta: 10 de julio de 2020].

CDN. El Comité de Derechos del Niño advierte sobre el grave efecto físico, emocional y psicológico de la pandemia Covid-19 en los niños y hace un llamado a los Estados para proteger los derechos de los niños [en línea]. Comité de Derechos del Niño, 2020. <https://tbinternet.ohchr.org/ Treaties/CRC/Shared\%20Documents/1_Global/INT_CRC_STA_9095_S. pdf $>$ [consulta: 10 de julio de 2020].

Cidh. Pandemia y Derechos Humanos en las Américas [en línea]. Comisión Interamericana de Derechos Humanos, resolución 1/2020. <http://www. oas.org/es/cidh/decisiones/pdf/Resolucion-1-20-es.pdf $>$ [consulta: 10 de julio de 2020].

Colmed. Género y salud en tiempos de Covid [en línea]. Colegio Médico de Chile, Departamento de Género y Salud, mayo de 2020. <http://www. colegiomedico.cl/wp-content/uploads/2020/05/Genero_COVID19_ compressed.pdf $>$ [consulta: 10 de julio de 2020].

El Mostrador. En un 500\% aumentan las denuncias por violencia intrafamiliar en Providencia durante la cuarentena [enlínea]. 14 de abril de 2020. < https:/ /www. elmostrador.cl/braga/2020/04/14/en-un-500-aumentan-las-denuncias-porviolencia-intrafamiliar-en-providencia-durante-la-cuarentena/ > [consulta: 10 de julio de 2020].

El Mostrador. 46\% más de carga laboral con teletrabajo: 92\% de las mujeres debe cocinar y limpiar mientras teletrabaja [en línea]. 20 de abril de 2020. <https:/ / www.elmostrador.cl/braga/2020/04/20/46-mas-de-carga-laboral-conteletrabajo-92-de-las-mujeres-debe-cocinar-y-limpiar-mientras-teletrabaja/ > [consulta: 10 de julio de 2020]. 
Foucault, Michel. Nacimiento de la biopolítica. Buenos Aires, Fondo de Cultura Económica, 2008.

Fuentes, Fernando. Después del Covid-19: la gente pide más democracia [en línea]. La Tercera, 17 de junio de 2020. <https://www.latercera.com/ mundo/noticia/despues-del-covid-19-la-gente-pide-mas-democracia/ RFYDZABIJ5ENJE4TIDE566PCGM/> [consulta: 10 de julio de 2020].

HRC. Ohchr \& Human Rights Committee Address Derogations During Covid-19. CCPR/C/128/2, 24 de abril de 2020, Human Rights Committee Statement on derogations from the Covenant in connection with the Covid-19 pandemic. <https://ijrcenter.org/2020/04/29/ohchr-human-rights-committeeaddress-derogations-during-covid-19/> [consulta: 10 de julio de 2020].

Hunter, Nan A. The Law of Emergencies: Public Health and Disaster Management. Oxford: Elsevier, 2009, https://doi.org/10.2202/1547-7355.1710.

INE. INE publica resultados de la Encuesta Nacional de Empleo del trimestre febrero-abril de 2020 [en línea]. Instituto Nacional de Estadística, Chile, 29 de mayo de 2020. <https://www.ine.cl/prensa/2020/05/29/ine-publicaresultados-de-la-encuesta-nacional-de-empleo-del-trimestre-febrero-abrilde-2020> [consulta: 10 de julio de 2020].

Ippf. Guía para proteger los derechos de mujeres y niñas durante la pandemia de Covid-19 [en línea]. Women's Link Worldwide, Amnistía International, Ippf. <https://www.womenslinkworldwide.org/files/3112/guia-para-protegerlos-derechos-de-mujeres-y-ninas-durante-la-pandemia-de-covid-19.pdf> [consulta: 10 de julio de 2020].

La Tercera. El detalle de los 46 proyectos que están en el Congreso y que el gobierno considera inconstitucionales [en línea]. 23 de junio de 2020. <https://www. latercera.com/politica/noticia/el-detalle-de-los-46-proyectos-que-estan-enel-congreso-y-que-el-gobierno-considera-inconstitucionales/74F3YLTNE5 FXTJEU3DH5R7MYNY/> [consulta: 10 de julio de 2020].

Mlambo-Ngcuka, Phumzile. Violencia contra las mujeres: la pandemia en la sombra [en línea]. ONU Mujeres, 6 de abril de 2020. <https://www.unwomen.org/ es/news/stories/2020/4/statement-ed-phumzile-violence-against-womenduring-pandemic $>$ [consulta: 10 de julio de 2020].

OEA. La Cidh llama a los Estados de la OEA a asegurar que las medidas de excepción adoptadas para hacer frente la pandemia Covid-19 sean compatibles con sus obligaciones internacionales [en línea]. Comunicado de prensa, 17 de abril de 2020. <http://www.oas.org/es/cidh/prensa/comunicados/2020/076.asp> [consulta: 10 de julio de 2020]. 
Olsen, Frances. El sexo del derecho. Traducción de Mariela Santero \& Christian Courtis. En: Kayris, David (ed.). The Politics of Law. New York, Pantheon, 1990. <http://www.derechoshumanos.unlp.edu.ar/assets/files/ documentos/el-sexo-del-derecho.pdf $>$ [consulta: 10 de julio de 2020].

ONU Mujeres. Covid-19 en América Latina y el Caribe: cómo incorporar a las mujeres y la igualdad de género en la gestión de la respuesta a la crisis [en línea]. ONU Mujeres América Latina y el Caribe, brief, 17 de marzo de 2020. <https://lac.unwomen.org/es/digiteca/publicaciones/2020/03/covidcomo-incorporar-a-las-mujeres-y-la-igualdad-de-genero-en-la-gestion-derespuesta $>$ [consulta: 10 de julio de 2020].

Ossul-Vermehren, Ignacia. Gender and disability in informal settlements during Covid-19: What we have learnt so far [en línea]. Blogs LSE, London School of Economics and Political Science, 29 de junio de 2020. < https://blogs.lse. ac.uk/gender/2020/06/29/gender-and-disability-in-informal-settlementsduring-covid-19-what-we-have-learnt-so-far/?fbclid=IwAR26oQfRDB 4xkQUKUeTJkS8N0UTRA7duOWRNGeEwURFmrskRzUI64fteazI> [consulta: 10 de julio de 2020].

Pérez Orozco, Amaya. Subversión feminista de la economía. Aportes para un debate sobre el conflicto capital-vida. España, Traficante de sueños, 2014. $<$ https:// www.traficantes.net/sites/default/files/pdfs/Subversi $\%$ C $3 \%$ B 3 n $\% 20$ feminista $\% 20$ de $\% 201 \mathrm{a} \% 20$ econom $\%$ C3 $\%$ ADa_Traficantes $\% 20 \mathrm{de} \% 20$ Sue $\%$ C3\%B1os.pdf $>$

Sepúlveda, Bárbara y Vivaldi, Lieta. Enfrentando la vulneración de los derechos de las mujeres y disidencia sexual: la experiencia de Abofem desde el 18 de octubre [en línea]. Pléyade, Número Especial, febrero de 2020. <http:// www.revistapleyade.cl/pleyade/ediciones/numero-revueltas-en-chile/ enfrentando-la-vulneracion-de-los-derechos-de-las-mujeres-y-disidenciasexual-la-experiencia-de-abofem-desde-el-18-de-octubre/> [consulta: 10 de julio de 2020]

Smart, Carol. The Woman of Legal Discourse. Social \& Legal Studies, vol. 1 (2002), 29-44. <https://doi.org/10.1177/096466399200100103> [consulta: 10 de julio de 2020].

Soy Chile. Amnistía Internacional por estados de excepción durante estallido: "Aumentó drásticamente el número de abusos cometidos por el Estado" [en línea]. 27 de febrero de 2020. <https://www.soychile.cl/Santiago/ Sociedad/2020/02/27/640645/Amnistia-Internacional-por-estados-deexcepcion-durante-estallido-aumento-drasticamente-el-numero-de-abusoscometidos-por-el-Estado.aspx> [consulta: 10 de julio de 2020]. 
UN. Thematic study on the issue of violence against women and girls and disability [en línea]. United Nations, General Assembly, 30 de marzo de 2012. <https:// www2.ohchr.org/english/issues/women/docs/A.HRC.20.5.pdf> [consulta: 10 de julio de 2020].

Undurraga, Verónica y Sadler, Michelle. The misrepresentation of conscientious objection as a new strategy of resistance to abortion decriminalization. Sexual and Reproductive Health Matters, vol. 27, 2019, issue 2, 17-19, DOI: 10.1080/26410397.2019.1610280. < https://www.tandfonline.com/doi/full /10.1080/26410397.2019.1610280> [consulta: 10 de julio de 2020].

Viera, Christian. Estado social como fórmula en la Constitución chilena [en línea]. Revista de Derecho Universidad Católica del Norte, vol. 21 núm. 2, Coquimbo, 2014. <https://scielo.conicyt.cl/scielo.php?script=sci_ arttext\&pid $=$ S071897532014000200014\&lng=es\&nrm=iso $>$ [consulta: 10 de julio de 2020].

Zúñiga, Yanira. La "genereización” de la ciudadanía. Apuntes sobre el rol del pensamiento sexual en el pensamiento feminista [en línea]. Revista de Derecho (Valdivia) v.22 n.2 Valdivia dic. 2009, 39-64, DOI: http://dx.doi. org/10.4067/S0718-09502009000200003. < https://scielo.conicyt.cl/scielo. php?script=sci_arttext\&pid=S0718-09502009000200003> [consulta: 10 de julio de 2020]. 